\title{
Projeto Idoso da Floresta: indicadores de saúde dos idosos inseridos na Estratégia de Saúde da Família (ESF-SUS) de Manaus-AM, Brasil
}

\author{
"Elderly from the Forest" Project: Health Indicators of Elderly's Family \\ Health Strategy in Manaus-AM's Health Districts, Brazil

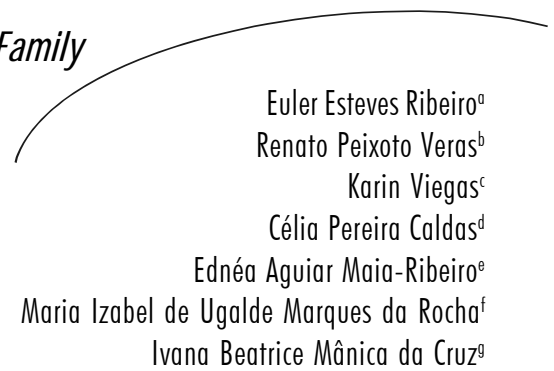

\section{Resumo}

Introdução: estudos sobre o idoso que vive na Região Norte do Brasil ainda são incipientes e necessários para subsidiar programas de saúde pública. Objetivos: o estudo aqui apresentado descreve o delineamento geral do Projeto Idoso da Floresta, analisando os principais indicadores de saúde de idosos inseridos no ESFSUS em Manaus-AM, distribuídos nos quatro distritos de saúde investigados. Metodologia: foi feita análise amostral por conglomerados em dois estágios: $\left(1^{\circ}\right)$ seleção das unidades básicas de saúde da família (UBS) como unidades de seleção; $\left(2^{\circ}\right)$ seleção do idoso como unidade amostral, escolhido randomicamente entre as famílias atendidas nas UBSs. Resultados: 1.509 idosos foram incluídos, sendo 810 (53,6\%) mulheres $(67,6 \pm 7,7$ anos) e 699 (46,4\%) homens com idade média de 66,8 6 ,9 anos. Em geral, o idoso investigado era casado/viúvo (94,8\%), com nenhuma ou baixíssima escolaridade $(46,5 \%)$, vivia em casas $(94,7 \%)$, recebia algum auxílio $(57,5 \%)$, era aposentado $(64,5 \%)$, sem renda ou recebia até um salário-mínimo $(67,9 \%)$. Destes, $85,5 \%$ eram autônomos e 10,3\% possuíam mais de quatro morbidades. Os indicadores tenderam a ser significativamente diferenciados conforme o distrito investigado. Conclusão: em geral, os perfis socioeconômico e cultural, da prevalência de morbidades e dependência tenderam a ser similares ao descrito em outros estudos brasileiros. Entretanto, existiram diferenças entre os distritos que devem ser consideradas nas estratégias de atenção e saúde do idoso. Análises complementares que estratifiquem a amostra por sexo, idade e perfil socioeconômico e cultural precisam ser conduzidas.

\author{
Palavras-chave: \\ Envelhecimento. \\ Saúde Pública. \\ Geriatria. Projetos \\ de Pesquisa. \\ Amostragem. Fatores \\ Socioeconômicos. \\ Prevalência. \\ Morbidade. Idoso \\ Fragilizado. \\ Manaus, AM
}

Universidade Estadual do Amazonas Universidade Aberta da Terceira Idade Manaus, AM, Brasil

Correspondência / Correspondence Euler Esteves Ribeiro UnATI-UEA

Av. Djalma Batista, 2470 - Chapada 69050-900 - Manaus, AM, Brasil E-mail: eer@vivax.com.br 


\section{Abstract}

Introduction: Studies on the elderly living in the Brazilian North Region are incipient and must be carried out to support health public policies. Objectives: this study describes the methodological design on the Projeto Idoso da Floresta, that analyzes the main health indicators of the elderly inserted in the Family Health Strategy, a Brazilian health public care program, in Manaus-AM. Methodology: a two-stage epidemiologic study was conducted as follows: (1st) selection of basic health units (UBS) as unit selection; (2nd) elderly selection as randomized sample unit from each UBS. Results: 1,509 elderly were included in the analysis, $810(53.6 \%)$

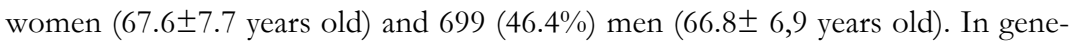
ral, the elderly investigated here were married/widowed $94,8 \%$ ), illiterate or with very low educational level $(46.5 \%)$, lived in houses $(94.7 \%)$, received some external support $(57.5 \%)$, retired $(64.5 \%)$, without or with lower economic income $(67.9 \%)$. From the sample analyzed, $85.5 \%$ were independent and $10.3 \%$ reported to have $\geq 4$ morbidities. These results tended to be different in Manaus's health districts. Conclusion: in general, the variables analyzed tended to be similar to results described in other Brazilian epidemiological studies. However, there are differences among districts that must be considered in the elderly's health care strategy. Complementary analysis of the data described here, with sex, age and socioeconomic stratification need to be performed.

\section{Key words:}

Aging. Public Health.

Geriatrics. Research

Design. Sampling

Studies. Socioeconomic Factors. Prevalence.

Morbidity. Frail Elderly. Manaus, city AM

\section{INTRODUÇÃOO}

No mesmo ano em que foi aprovada a Política Nacional do Idoso, o Ministério da Saúde (PNI/Lei no 8.842/94) ${ }^{1}$ assumiu a implantação de um novo modelo assistencial de saúde, inicialmente conhecido como Programa de Saúde da Família (PSF) e posteriormente denominado Estratégia de Saúde da Família, com o objetivo de reverter o modelo assistencial baseado na prática hospitalar individualista de baixa resolução. Esta mudança de paradigma é crucial para o cuidado da saúde geral, em especial dos idosos.

A falta de conhecimento sobre o idoso brasileiro, a escassez de recursos humanos com competência para assisti-lo e a grande varia- ção nas taxas de envelhecimento populacional nas diferentes regiões brasileiras fazem com que a transposição das disposições do Projeto de Lei para o cotidiano da população seja um dos grandes desafios dos próximos 20 anos e dependa do contexto regional. Considerando as diferenças geográficas brasileiras em relação ao envelhecimento, a proporção dos idosos é muito grande nas regiões mais ao sul do país, enquanto que a Região Norte ainda é aquela em que ocorre um número mais reduzido de idosos. ${ }^{2}$ Tais diferenças são reflexos das variações sociodemográficas, étnicas e ambientais entre as regiões brasileiras.

Entretanto, com o conhecimento produzido no mundo e no Brasil, as regiões e estados que ainda hoje apresentam um processo 
de envelhecimento menos acentuado poderão se beneficiar, com a perspectiva de desenvolver estratégias de apoio ao idoso que não foram ou puderam ser implantadas nas regiões onde o envelhecimento populacional foi mais acelerado. No caso do Amazonas, existe a real possibilidade de implantação de um programa estratégico pró-envelhecimento bem-sucedido que seja de alto impacto econômico e social, e influencie a própria qualidade de vida do indivíduo e da sociedade amazonense do futuro. Isto porque a velhice, em um contexto apropriado, como ocorreu em países desenvolvidos que tiveram um tempo mais longo no seu processo de envelhecimento, "passa a ser encarada como fase normal da vida e não como marginal'. 3

Ainda assim, a diversidade sociocultural, econômica, étnica e macroambiental impõem a necessidade de estudos epidemiológicos sobre o idoso que vive nas áreas tropicais amazônicas. Esta idéia está subsidiada pela natureza multifatorial e complexa do fenômeno do envelhecimento biológico, o que faz com que seja muito difícil que todas as variáveis que incidem sobre tal fenômeno e sobre a etiologia das doenças associadas à idade sejam investigadas ao mesmo tempo e sejam similares em todo o mundo. ${ }^{4}$

Adicionalmente, com o aumento no número de idosos na população, há profunda necessidade de capacitar recursos humanos aptos a manejar os indivíduos pertencentes a este grupo etário, que são altamente suscetíveis à fragilidade. Mota e Aguiar, ${ }^{5}$ a este respeito, comentam: (...) A população idosa experi- menta alteracões fisiológicas e patológicas que cursam com crescente dependência. Do ponto de vista teórico, tal fato demanda aprofundamento de conceitos, tais como niveis de prevenção, paliativismo, suporte e apoio social. Na prática, aos profissionais cabe trabalhar compacientes e suas familias/cuidadores, para enfrentando juntos situações de perda e morte. (...) Intervencooes baseadas em tal moldura extrapolam o modelo biomédico, hegemônico. Enfocando o estudo da doença, o modelo biomédico secundariza aspectos sociais, econômicos e subjetivos na determinação do processo saúdedoença e caracteriza-se pelo reducionismo, ao tratar fenômenos complexos como princípios primários simples, epelo dualismo mente-corpo."

Como o estágio ontogenético marcado pela velhice é altamente complexo, o idoso não se enquadra nesse modelo reducionista, já que o mesmo não oferece condições de estabelecer, para além do processo curativo da doença, uma rede de cuidados sociais, econômicos e individualmente benéficos. Silvestre, ${ }^{6}$ em estudo conduzido no Rio Grande do Sul, observou que os idosos internados em hospitais gerais não recebiam assistência médica e psicossocial de acordo com suas necessidades: problemas típicos da idade não foram identificados e tratados adequadamente. ${ }^{6}$ As conseqüências disto incluem, assim, o prolongamento no tempo da internação, redução na capacidade funcional do idoso por ocasião da alta e reinternações freqüentes.

Portanto, o aumento no número de idosos na população acarreta crescimento das despesas com tratamentos médicos e hospitalares, ao mesmo tempo que representa um desafio para as autoridades sanitárias, especi- 
almente no que tange à implantação de novos modelos e métodos de planejamento, gerência e prestação de cuidados. ${ }^{7}$ Isto porque, em geral, as doenças dos idosos são crônicas e múltiplas, perduram por vários anos e exigem acompanhamento médico constante $\mathrm{e}$ medicação contínua. Além disso, a abordagem médica tradicional, focada em uma queixa principal, e o hábito do médico de reunir as queixas e os sinais em um único diagnóstico, podem ser adequados ao adulto jovem, mas não ao idoso. ${ }^{8}$

Levando em conta esse panorama geral, foi implantado em 2007 um estudo populacional dos idosos incluídos na Estratégia de Saúde da Família do Sistema Único de Saúde (ESF-SUS) do município de Manaus, genericamente denominado Projeto Idoso da Floresta, que busca estabelecer as principais características sócio-epidemiológicas, biológicas e de qualidade de vida do "idoso que vive na floresta tropical amazônica". O estudo concentrou-se nos idosos que freqüentam o ESF-SUS e não o idoso em geral que vive em tal município, uma vez que tem como foco estratégico entender quais são as reais demandas sociais e de saúde que hoje se apresentam aos serviços de saúde que manejam esses idosos.

Em 1999, o ESF-SUS passou a ser considerado pelo Ministério da Saúde uma estratégia estruturante dos sistemas municipais de saúde, com vistas a reorientar o modelo assistencial e imprimir nova dinâmica na organização dos serviços e ações de saúde. $\mathrm{O}$ modelo preconiza uma equipe de saúde da família de caráter multiprofissional (médico generalista, enfermeiro, auxiliar de enfermagem e agente comunitário de saúde), com definição de território de abrangência, adscrição de clientela, cadastramento e acompanhamento da população residente na área. Tem como objetivo que a unidade de saúde da família constitua a porta de entrada ao sistema local e o primeiro nível de atenção, o que supõe a integração à rede de serviços mais complexos. A ESF-SUS recomenda que cada equipe fique responsável entre $600 \mathrm{a}$ mil famílias, o que representa 2.400 a 4.500 habitantes. ${ }^{9}$ Deste modo, estudos associados ao ESF-SUS incidem sobre um número potencialmente alto de indivíduos da população.

Adicionalmente, ainda que o Projeto Idoso da Floresta esteja científica e epidemiologicamente delineado, sua execução está fortemente apoiada na formação de recursos humanos em nível de pós-graduação na área gerontológica que trabalham com idosos nas redes municipais e estaduais de saúde. Acredita-se que a relevância do Projeto Idoso da Floresta esteja no seu delineamento, que preconiza uma associação integradora do trinômio educação-pesquisa-ação nos serviços de saúde. Os recursos humanos formados, de modo concomitante, se educam na área gerontológica, se capacitam na área da execução de estudos científicos e no uso de instrumentos e metodologia científicas, e trabalham diretamente dentro da sua realidade local, a partir dos desafios e questionamentos impostos pela própria realidade. Assim, neste estudo se apresentam o delineamento e o perfil geral social e de saúde do idoso 
incluído no ESF-SUS do município de Manaus, que é a população-alvo do Projeto Idoso da Floresta.

\section{METODOLOGIA}

Área e características gerais do município e de sua população

O Projeto Idoso da Floresta foi implantado a partir de outubro de 2007 no município de Manaus, Estado do Amazonas, e suas características gerais são comentadas a seguir. $\mathrm{O}$ município está situado na confluência dos rios Negro e Solimões. A população de Manaus, em 2007, foi estimada em 1.646.602 habitantes, conforme contagem realizada pelo Instituto Brasileiro de Geografia e Estatística (IBGE), sendo a oitava cidade brasileira mais populosa e a maior da Região Norte do país. Geograficamente, é caracterizada por planícies, baixo planaltos, terras firmes e tem altitude média inferior a 100 metros. O clima é equatorial, com umidade relativa elevada durante o ano $(80-$ $90 \%$ e e média térmica anual de $28^{\circ} \mathrm{C}$, oscilando de $14^{\circ} \mathrm{C}$ a $40^{\circ} \mathrm{C}$. A vegetação é densa e tipicamente coberta pela Floresta Amazônica.

A maior parte da população está concentrada nas zonas leste e oeste. Os principais bairros que compõem o município de $\mathrm{Ma}$ naus por zona são: Zona Norte: Cidade Nova (215.449 habitantes), Nova Cidade (31.443 habitantes); Zona Leste: São José (188.670 habitantes), Jorge Teixeira (116.677 habitantes), Amazonino Mendes (53.839 habitantes), Tancredo Neves (42.092), Zumbi (32.604 habitantes); Zona Oeste e Centro-Oeste:
Compensa (71.013 habitantes), Alvorada (64.222); Zona Sul e Centro-Sul: Parque Dez (69.407 habitantes), Japiim (58.616 habitantes), Coroado (55.495 habitantes), Petrópolis (44.610 habitantes) e Flores (34.406 habitantes). A densidade demográfica está calculada em 144 habitantes por $\mathrm{Km}^{2}$.

Estruturalmente, $77 \%$ do município são atendidos pela rede de distribuição de energia elétrica, $65 \%$ pela rede de esgoto, $86 \%$ pela coleta de lixo e $69 \%$ pelo abastecimento de água. A taxa de mortalidade infantil até cinco anos de idade é de 21,26 a cada mil crianças, a taxa de fecundidade é de 3,74 filhos por mulher e a taxa de alfabetização é de $94,63 \%$. O cálculo do índice de desenvolvimento humano (IDH) é de 0,788 , o que representa um desenvolvimento moderado. A esperança de vida ao nascer é superior a 67,7 anos, baixa em relação à esperança de vida média do país, que é de aproximadamente 71 anos de idade.

Para fins de estudo sobre envelhecimento, é importante caracterizar o perfil étnico da população investigada. A formação histórica de Manaus é representada pela alta miscigenação entre as três etnias consideradas básicas da população brasileira: indígena, européia (maioria de origem portuguesa) e africana. Posteriormente a sua fundação e ao primeiro período de sua história, a cidade recebeu imigrantes que também contribuíram etnicamente, em especial árabes, judeus e japoneses.

\section{Delineamento e população do estudo}

O estudo foi delineado de forma a produzir estimativas para os idosos incluídos na 
ESF-SUS do município de Manaus, distribuídos nos quatro distritos de saúde do município (norte, sul, leste e oeste). Segundo dados da Secretaria Municipal da Saúde e do estudo realizado por Escorel et al., ${ }^{10} \mathrm{O}$ ano de implantação do ESF-SUS no município foi 1999, e até 2007 lá havia 171 equipes de saúde da família, representando uma coberta de $41 \%$ da população. Estima-se, assim, que o ESF-SUS acompanhou, em 2007, 675.107 pessoas e que, destas, 33.080 eram idosos com 60 ou mais anos de idade. No momento do estudo, apenas 169 unidades estavam efetivamente funcionando no ano de 2007, segundo informações da Secretaria Municipal de Saúde.

A definição do tamanho amostral (1.680 idosos) baseou-se na experiência de pesquisas semelhantes, desenvolvidas em outros locais, e considerou a possibilidade de perda na ordem de $20 \%$. O estudo foi realizado a partir de uma amostra por conglomerados em dois estágios:

$1^{\text {o }}$ Estágio - as unidades de saúde da família foram selecionadas como unidades básicas de seleção. Nestas unidades, distribuídas nas quatro zonas geográficas de $\mathrm{Ma}$ naus, os agentes de saúde da família foram utilizados como unidades primárias de seleção. Os 70 entrevistadores foram inicialmente divididos em cinco grupos. Para cada grupo foram sorteadas as unidades onde seriam coletadas as informações. Cada entrevistador visitou duas unidades, perfazendo um total de 140 unidades básicas de saúde incluídas no estudo, o que representa $83 \%$ das mesmas. Conhecidas as unidades do estudo, o número e a identificação dos agentes de saúde foram arrolados, ocorrendo novo sorteio para identificar o agente de saúde da família que iria acompanhar e identificar os idosos participantes da pesquisa, também de modo randomizado. Os procedimentos de organização geral da infra-estrutura da pesquisa, bem como os sorteios foram coordenados pela equipe de profissionais da UnATI-UEA.

$2^{\mathbf{o}}$ Estágio - o idoso foi considerado a unidade amostral, também sendo escolhido randomicamente a partir do cadastro das famílias atendidas pelo agente de saúde. Os dados foram coletados no domícilio do entrevistado, por meio de entrevista estruturada. Avaliação antropométrica foi realizada na própria casa do idoso, pelos entrevistadores, que receberam um conjunto de equipamentos portáteis de avaliação do peso corporal, circunferência abdominal e altura. A avaliação dos indicadores bioquímicos foi realizada através do encaminhamento do voluntário para a unidade básica de saúde que procedeu à coleta dos dados orientada pelo entrevistador. Em caso de impossibilidade de deslocamento do idoso, a coleta foi realizada no domicílio do mesmo, através da estrutura da rede de saúde municipal. Todos os entrevistadores possuíam nível educacional universitário e eram discentes do $1^{\circ}$ Curso de Especialização em Gerontologia e Saúde do Idoso, coordenado pela Universidade Aberta da Terceira Idade, da Universidade do Estado do Amazonas (UnATI/UEA), implantado a partir de março de 2007. A meta de inclusão de idosos de cada entrevistador foi estimada em 24 idosos, com uma proporção de gêne- 
ro próxima de 1:1. Neste caso, não foi obedecida a proporção da distribuição dos sexos na faixa etária igual e superior a 60 anos, que é de 3:1 (três mulheres/um homem), com o propósito de aumentar o número amostral deste grupo populacional, minimizando o impacto de amostras relativamente baixas de indivíduos homens nos estudos gerontológicos. Caso a proporção populacional fosse mantida, análises estratificadas da saúde do idoso homem poderiam ficar comprometidas. Por este motivo, neste trabalho a caracterização e os cálculos de prevalência de morbidades e outros indicadores são gênero-dependentes e não são representativos da população idosa em geral, mas de grande interesse para a ESF-SUS de Manaus-AM.

O estudo foi aprovado pelo Comitê de Ética em Pesquisa da Universidade do Estado do Amazonas (UEA) e conduzido segundo as normativas da Resolução no 196/1996 da CONEP. Todos os idosos assinaram termo de consentimento livre e esclarecido.

\section{Variável dependente}

A variável dependente do estudo foi o local geográfico de inserção do idoso na ESFSUS, identificado através das quatro grandes zonas de Manaus-AM (norte, sul, leste e oeste). Deste modo, as variáveis independentes foram comparadas entre tais zonas. Esta primeira informação é relevante para a rede pública de saúde, porque serve tanto para caracterizar de modo geral o idoso que está sendo atendido pelo ESF-SUS de Manaus, quanto para identificar possíveis variações relacionadas com o local de moradia e, portanto, com unidades básicas de saúde específicas. Para conduzir esta fase do estudo, utilizou-se entrevista estruturada aplicada pelos entrevistadores.

\section{Variáveis independentes}

O Projeto Idoso da Floresta, na sua primeira fase, irá investigar sete grandes grupos de características do idoso: características sociodemográficas e de ocupação e renda; composição familiar e relações; perfil de saúde (através da avaliação de morbidades crônicas não-transmissíveis, morbidades infecto-contagiosas no último ano e imunização) e estilo de vida; atividades de vida diária e nível de dependência; qualidade de vida; capacidades motivacionais e autocuidado; indicadores de equilíbrio e detecção da osteoporose; indicadores psicogeriátricos.

Entretanto, este estudo analisou apenas os indicadores socioeconômicos, culturais, de dependência e de morbidade geral por zona geográfica de inserção do idoso no ESF-SUS. As variáveis socioeconômicas demográficas investigadas foram: escolaridade, situação conjugal, renda pessoal mensal (em salários mínimos nacionais vigentes à época da entrevista), tipo de moradia (casa, apartamento, palafita e barraco), recepção de auxílio (dinheiro, alimentação, vestuário, saúde, habitação), nível de autonomia e presença de morbidades.

A autonomia foi analisada através do índice de Barthel, ${ }^{11}$ instrumento que avalia o nível de independência do sujeito para a realização de dez atividades básicas da vida: alimentação, 
higiene pessoal, utilização de sanitários, banharse, vestir-se e despir-se sem auxílio de outra pessoa, controle dos esfíncteres, deambulação, transferência da cadeira para a cama, subir e descer escada. A pontuação da escala varia de 0-100, sendo que zero corresponde à máxima dependência para todas as atividades de vida diária (AVD) e 100 equivale à independência total. A partir do relato de história prévia para 12 morbidades crônicas não-transmissíveis, os idosos foram categorizados naqueles que autorelataram estar livres destas morbidades, possuírem uma, duas, três a quatro ou mais de quatro morbidades.

\section{Análise dos dados}

Os dados foram inicialmente digitados em planilha eletrônica Excel pelos próprios entrevistadores do estudo, que receberam capacitação conjunta para a execução de tal procedimento. Posteriormente, a checagem da qualidade dos dados digitados foi realizada pela equipe de apoio à pesquisa da UnATI-UEA, dos pesquisadores principais do estudo, e os dados foram então analisados, utilizando-se o programa estatístico SPSS versão 12.0 .

Os resultados gerais foram expressos em média e desvio padrão. A comparação entre os indicadores gerais sociodemográficos e de saúde dos idosos participantes do estudo foi feita entre os gêneros e as zonas geográficas. A análise univariada incluiu comparação das variáveis categóricas por qui-quadrado e das variáveis quantitativas por $t$ de Student. Todas as comparações com valores alfa de $p<0,05$ foram consideradas significativas.

\section{RESULTADOS}

Um total de 1.509 idosos foi incluído no estudo, representando 89,8\% da amostra inicial estimada. Da amostra estimada, 171 idosos não foram incluídos nas análises porque sete unidades básicas de saúde estavam em reforma e sem condições de participar do estudo, o que determinou a exclusão de 168 voluntários. Três idosos não participaram porque não foi possível obter as informações da pesquisa junto a eles e seus cuidadores. Os idosos estudados apresentavam as seguintes características: 53,6\% eram do sexo feminino

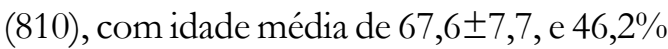
eram do sexo masculino (699), com idade média de 66,8 0,9 anos.

A distribuição dos idosos por zona geográfica foi a seguinte: zona norte de 311 (20,6\%); zona sul 513 (34,0\%); zona leste 253 $(16,8 \%)$; e zona oeste $432(28,6 \%)$. Na zona norte, $136(43,7 \%)$ eram homens e $175(53,3 \%)$ mulheres; na zona sul $262(56,3 \%)$ eram mulheres e 251 (48,9\%) eram homens; na zona leste $104(41,1 \%)$ eram homens e $149(58,9 \%)$ mulheres; e na zona oeste 208 (48,1\%) eram homens e 224 (51,9\%) eram mulheres. Estas diferenças não foram estatisticamente significativas $(p=0,221)$.

As variáveis sociodemográficas, econômicas e os indicadores de saúde e de dependência foram analisados e comparados entre os quatro distritos de saúde. Os resultados são apresentados nas tabelas 1 e 2 . 
Tabela 1 - Indicadores sócio-demográficos, econômicas e de dependência dos idosos inseridos na Estratégia de Saúde da Família (ESF-SUS) do Município de Manaus-AM por zona geográfica de residência, Manaus, AM, 2008.

\begin{tabular}{|c|c|c|c|c|c|c|}
\hline Indicadores & $\begin{array}{l}\text { Norte } \\
\mathrm{n}(\%)\end{array}$ & $\begin{array}{c}\text { Sul } \\
\mathrm{n}(\%)\end{array}$ & $\begin{array}{l}\text { Leste } \\
\mathrm{n}(\%)\end{array}$ & $\begin{array}{l}\text { Oeste } \\
\text { n (\%) }\end{array}$ & $\begin{array}{l}\text { Total } \\
\mathrm{n}(\%)\end{array}$ & $\mathrm{p}$ \\
\hline Situação conjugal & & & & & & $<0,001$ \\
\hline Solteiro & $16(5,1)$ & $51(9,9)$ & $43(17,0)$ & $30(6,9)$ & $140(9,3)$ & \\
\hline Casado $^{1}$ & $189(60,8)$ & $281(54,8)$ & $111(43,9)$ & $263(60,9)$ & $844(66,9)$ & \\
\hline Viúvo & $83(26,7)$ & $146(28,5)$ & $79(31,2)$ & $113(26,2)$ & $421(27,9)$ & \\
\hline Divorciados $^{2}$ & $23(7,4)$ & $35(6,8)$ & $20(7,9)$ & $26(6,0)$ & $104(6,9)$ & \\
\hline Escolaridade & & & & & & $<0,001^{4}$ \\
\hline Analfabeto & $121(39,5)$ & $157(31,0)$ & $118(47,4)$ & $145(34,1)$ & $541(34,1)$ & \\
\hline Alfabetizado ${ }^{3}$ & $55(18,0)$ & $61(12,0)$ & $40(12,0)$ & $39(9,2)$ & $185(12,4)$ & \\
\hline Primário incompleto & $64(20,9)$ & $121(23,9)$ & $55(22,1)$ & $103(24,2)$ & $343(23,1)$ & \\
\hline Primário completo & $30(9,8)$ & $102(20,1)$ & $26(10,4)$ & $96(22,6)$ & $254(17,1)$ & \\
\hline Ginasial incompleto & $20(6.5)$ & $32(6.3)$ & $10(4.0)$ & $14(3.3)$ & $76(5,1)$ & \\
\hline Ginasial completo & $10(3,3)$ & $20(3,9)$ & $06(2,4)$ & $10(2,4)$ & $46(3,1)$ & \\
\hline Secundário incompleto & $02(0,7)$ & $1(0,2)$ & $0(0)$ & $3(0.7)$ & $06(0,4)$ & \\
\hline Secundário completo & $02(0,7)$ & $08(1,6)$ & $03(1,2)$ & $05(1,2)$ & $18(1,2)$ & \\
\hline Superior incompleto & $0(0)$ & $02(0,7)$ & $0(0)$ & $03(0,4)$ & $05(0,3)$ & \\
\hline Superior completo & $0(0)$ & $03(0,6)$ & $0(0)$ & $02(0,5)$ & $05(0,3)$ & \\
\hline Não sabia informar & $02(0,7)$ & $0(0)$ & $01(0,4)$ & $05(1,2)$ & $08(0,5)$ & \\
\hline Tipo de moradia & & & & & & 0,212 \\
\hline Casa & $301(96,8)$ & $490(95,5)$ & $238(94,1)$ & $400(92,6)$ & $1429(94,7)$ & \\
\hline Apartamento & $01(0,3)$ & $03(0,6)$ & $01(0,4)$ & $04(0,9)$ & $09(0,6)$ & \\
\hline Quarto/Cômodo & $08(2,6)$ & $09(1,8)$ & $10(4,0)$ & $18(4,2)$ & $45(3,0)$ & \\
\hline Barraco & $01(0,3)$ & $03(0,6)$ & $02(0,8)$ & $06(1,4)$ & $12(0,8)$ & \\
\hline Palafita & $0(0)$ & $08(1,6)$ & $02(0,8)$ & $04(0,9)$ & $14(0,9)$ & \\
\hline Recebe auxilio financeiro & & & & & & $<0,001$ \\
\hline Recebe & $201(65,5)$ & $303(60,6)$ & $108(44,4)$ & $232(55,4)$ & $844(57,5)$ & \\
\hline Não recebe & $104(33,9)$ & $192(38,4)$ & $135(55,6)$ & $185(44,2)$ & $616(41,9)$ & \\
\hline Não respondeu & $02(0,7)$ & $04(0,8)$ & $0(0)$ & $02,(0,5)$ & $08(0,5)$ & \\
\hline Não sabe & $0(0)$ & $01(0,2)$ & $0(0)$ & $(0)$ & 01 & \\
\hline Tipo de auxilio recebido & & & & & & 0,02 \\
\hline Dinheiro & $140(85,4)$ & $235(86,4)$ & $108(80)$ & $174(74,4)$ & $657(81,6)$ & \\
\hline Vestuário & $06(3,7)$ & $12(4,4)$ & $03(2,2)$ & $11(4,7)$ & $32(4,0)$ & \\
\hline Saúde & $0(0)$ & $10(3,7)$ & $5,9(08)$ & $098(3,4)$ & $26(3,2)$ & \\
\hline Habitação & $0(0)$ & $02(0,7)$ & $01(0,7)$ & $04(1,7)$ & $07(0,9)$ & \\
\hline Alimentação & $09(5,5)$ & $04(1,5)$ & $09(6,7)$ & $23(9,8)$ & $45(5,6)$ & \\
\hline Mais de um tipo de auxilio & $09(5,5)$ & $09(3,3)$ & $06(4,4)$ & $14(6,0)$ & $38(4,7)$ & \\
\hline \multicolumn{7}{|l|}{ Ocupação } \\
\hline Aposentado & $199(64,0)$ & $329(64,1)$ & $167(66,0)$ & $278(64,4)$ & $973(64,5)$ & 0,905 \\
\hline Ativo & $22(7,1)$ & $35(6,8)$ & $18(7,1)$ & $22(5,1)$ & $97(6,4)$ & \\
\hline Dona de Casa & $39(12,5)$ & $68(13,3)$ & $36(14,2)$ & $58(13,4)$ & $201(13,3)$ & \\
\hline Pensionista & $28(9,0)$ & $47(9,2)$ & $19(7,5)$ & $35(8,1)$ & $129(8,5)$ & \\
\hline Desempregado & $16(5,1)$ & $20(3,9)$ & $06(2,4)$ & $22(5,1)$ & $64(4,2)$ & \\
\hline Não respondeu & $07(2,3)$ & $14(2,7)$ & $07(2,8)$ & $17(3,9)$ & $45(3,0)$ & \\
\hline Renda mensal & & & & & & $<0,001$ \\
\hline Sem renda própria & $33(10,6)$ & $53(10,3)$ & $29(11,5)$ & $38(8,8)$ & $153(10,1)$ & \\
\hline 1 salário & $199(64)$ & $250(48,7)$ & $163(64,4)$ & $259(60,0)$ & $871(57,7)$ & \\
\hline 1 a 3 salários & $59(19,0)$ & $155(30,2)$ & $45(17,8)$ & $97(22,5)$ & $356(23,6)$ & \\
\hline$>3$ salários & $11(3,5)$ & $41(8,0)$ & $07(2,8)$ & $26(6,0)$ & $85(5,6)$ & \\
\hline Não respondeu & $07(2,3)$ & $14(2,7)$ & $08(3,2)$ & $12(2,8)$ & $41(2,7)$ & \\
\hline Não sabe & $02(0,6)$ & $0(0)$ & $01(0,4)$ & $0(0)$ & $3(0,2)$ & \\
\hline
\end{tabular}

$\mathrm{N}=$ tamanho amostral; ${ }^{1}$ inclui também os idosos que relataram ter união estável; ${ }^{2}$ inclui também os separados, desquitados; 3 alfabetizados fora da escola formal; ${ }^{4}$ como o número de idosos com escolaridade superior ao ginasial completo foi muito baixa, para fins de análise estatísticas as categorias de escolaridade secundaria e superior foram agrupadas. ${ }^{5}$ como o número idosos com dependência severa e total foi baixo, os mesmos foram agrupados para fins de análise estatística. Comparações estatísticas por teste Qui-quadrado. 
Tabela 2 - Indicadores de cuidado com a saúde, morbidades e de dependência dos idosos inseridos na Estratégia de Saúde da Família (ESF-SUS) do Município de Manaus-AM por distrito de saúde. Manaus, AM, 2008.

\begin{tabular}{|c|c|c|c|c|c|c|}
\hline Indicadores & $\begin{array}{l}\text { Norte } \\
\mathrm{n}(\%)\end{array}$ & $\begin{array}{l}\text { Sul } \\
\mathrm{n}(\%)\end{array}$ & $\begin{array}{l}\text { Leste } \\
\mathrm{n}(\%)\end{array}$ & $\begin{array}{l}\text { Oeste } \\
\mathrm{n}(\%)\end{array}$ & $\begin{array}{l}\text { Total } \\
\mathrm{n}(\%)\end{array}$ & $p$ \\
\hline $\begin{array}{r}\text { Consulta ginecologista pós- } \\
\text { menopausa }\end{array}$ & $112(65,1)$ & $163(62,5)$ & $73(49,3)$ & $115(53,5)$ & $463(58,2)$ & 0,05 \\
\hline Mamografia & $81(46,3)$ & $100(39,1)$ & $49(33,1)$ & $52(24,3)$ & $282(35,6)$ & 0,02 \\
\hline Reumatismo & $80(25,7)$ & $166(32,4)$ & $59(23,3)$ & $121(28,0)$ & $426(28,2)$ & 0,04 \\
\hline Hipertensão & $162(52,1)$ & $333(64,9)$ & $135(53,4)$ & $232(53,7)$ & $862(57,1)$ & $\leq 0,001$ \\
\hline DAC & $28(9,0)$ & $71(14,8)$ & $26(10,3)$ & $53(12,3)$ & $178(11,8)$ & 0,168 \\
\hline Diabetes Mellitus 2 & $69(22,2)$ & $125(24,4)$ & $62(24,5)$ & $100(23,1)$ & $356(23,6)$ & 0,879 \\
\hline AVC & $16(5,1)$ & $29(5,7)$ & $11(4,3)$ & $27(6,3)$ & $83(5,5)$ & 0,750 \\
\hline Distúrbios estomacais & $15(4,8)$ & $17(3,3)$ & $12(4,7)$ & $33(7,6)$ & $77(5,1)$ & 0,026 \\
\hline Osteoporose & $13(4,2)$ & $32(6,2)$ & $13(5,1)$ & $38(8,8)$ & $96(6,4)$ & 0,06 \\
\hline Câncer & $08(2,6)$ & $9(1,8)$ & $4(1,6)$ & $13(3,0)$ & $34(2,3)$ & 0,500 \\
\hline Obesidade* & $54(18,4)$ & $90(17,8)$ & $33(13,1)$ & $100(23,5)$ & $280(18,8)$ & 0,002 \\
\hline Desnutrição & $11(3,6)$ & $15(3,0)$ & $06(2,4)$ & $18(4,2)$ & $50(3,4)$ & \\
\hline Tabagismo & $90(41,7)$ & $126(42,4)$ & $43(36,1)$ & $114(44,0)$ & $373(41,9)$ & 0,329 \\
\hline Internação hospitalar** & $51(16,4)$ & $84(16,4)$ & $39(15,4)$ & $66(15,3)$ & $240(15,90)$ & 0,957 \\
\hline \multicolumn{7}{|l|}{ Imunizações } \\
\hline $\begin{array}{r}\text { Anti-tetânica }(<10 \\
\text { anos })\end{array}$ & $231(74,3)$ & $215(41,9)$ & $132(52,2)$ & $237(54,9)$ & $815(54,0)$ & $\leq 0,001$ \\
\hline Gripe (anual) & $270(86,8)$ & $337(65,7)$ & $186(73,5)$ & $324(75,0)$ & $1117(74,0)$ & $\leq 0,001$ \\
\hline $\begin{array}{r}\text { Anti- } \\
\text { pneumocócica } \\
(<5 \text { anos })\end{array}$ & $41(13,2)$ & $53(10,3)$ & $31(12,3)$ & $16(3,7)$ & $141(9,3)$ & $\leq 0,001$ \\
\hline Quedas no último ano & $50(16,2)$ & $80(17,6)$ & $52(24,0)$ & $100(23,6)$ & $282(20,1)$ & 0,007 \\
\hline Queda com fratura & $03(1,5)$ & $13(4,5)$ & $03(2,0)$ & $15(6,5)$ & $34(3,9)$ & 0,086 \\
\hline Autonomia & & & & & & $0,111^{5}$ \\
\hline Independente & $214(77,3)$ & $389(76,6)$ & $188(75,2)$ & $321(74,7)$ & $1112(75,9)$ & \\
\hline Dependência leve & $28(10,1)$ & $55(10,8)$ & $19(7,6)$ & $39(9,1)$ & $141(9,6)$ & \\
\hline $\begin{array}{r}\text { Dependência } \\
\text { moderada }\end{array}$ & $28(10,1)$ & $50(9,8)$ & $33(13,2)$ & $65(15,1)$ & $176(12,0)$ & \\
\hline $\begin{array}{r}\text { Dependência } \\
\text { severa }\end{array}$ & $07(2,5)$ & $12(2,4)$ & $06(2,4)$ & $05(1,2)$ & $30(2,0)$ & \\
\hline Dependência total & $0(0)$ & $02(0,4)$ & $04(1,6)$ & $0(0)$ & $6(0,4)$ & \\
\hline
\end{tabular}

$\mathrm{n}=$ tamanho amostral; comparações estatísticas por teste Qui-quadrado; * obesidade/desnutrição foram avaliadas em 1492 indivíduos e incluídas no mesmo teste estatístico; Obesidade $\left(\geq 30 \mathrm{~kg} / \mathrm{m}^{2}\right)$, desnutrição $\left(\leq 18,5 \mathrm{~kg} / \mathrm{m}^{2}\right), \mathrm{DAC}=\mathrm{doença} \mathrm{arterial}$ coronariana; $\mathrm{AVC}=$ acidente vaso cerebral **Internação hospitalar no último ano. 
Considerando-se o conjunto total da amostra os idosos inseridos no ESF-SUS do município de Manaus, os resultados mostraram que esse idoso em média é casado ou viúvo $(94,8 \%)$, com nenhuma ou baixíssima escolaridade (46,5\%), vive em casas $(94,7 \%)$, recebe auxílio $(57,5 \%)$ geralmente monetário (81,6\%), é aposentado $(64,5)$, não tem renda ou recebe até um salário-mínimo $(67,9 \%)$.

Quando a análise foi estratificada pelos distritos de saúde identificados através das quatro zonas geográficas, foram observadas diferenças significativas na grande parte das variáveis. No caso, a zona leste apresentou um número de idosos solteiros, uma freqüência alta de indivíduos analfabetos e que também relataram receber menos auxílios do que os idosos das outras zonas do município. Juntamente com os da zona norte, os idosos da zona leste apresentaram menor renda mensal. Por outro lado, a zona sul foi a que apresentou idosos que declararam renda mensal mais elevada. Similarmente à zona oeste, a zona sul também apresentou idosos com maior nível de escolaridade.

Em relação aos indicadores de saúde e dependência, 85,5\% dos idosos foram considerados autônomos pelo índice de Barthel, ${ }^{11}$ $74 \%$ relataram ter sido imunizados para a gripe nos últimos 12 meses, 14\% relataram não possuir nenhuma doença crônica não-transmissível que fosse do seu conhecimento, 215 $(14,3 \%)$ não relataram nenhum tipo de morbidade crônica que foi questionada, 373 $(24,7 \%)$ relataram uma morbidade, 765
$(50,6 \%)$ relataram possuir de duas a quatro morbidades e $155(10,3 \%)$ mais de quatro morbidades.

As mulheres que apresentaram maior prevalência de cuidado com a saúde ginecológica, avaliada através da consulta anual ao ginecologista, realização de exame citopatológico de colo do útero e mamografia foram as inseridas no ESF-SUS das zonas norte e sul. Entretanto, também foi na zona sul que se observou a maior prevalência de hipertensão arterial sistêmica.

Em relação às imunizações, as mesmas foram significativamente maiores, tanto para a vacina antitetânica quanto para a gripe, na zona norte. As prevalências de diabetes mellitus, hipertensão arterial sistêmica, doença arterial coronariana, acidente vasoencefálico, osteoporose e câncer, bem como a freqüência anual de internações hospitalares, foram similares para todos os idosos investigados. Já a prevalência de reumatismo foi maior em idosos da zona sul e oeste, e os distúrbios gastrointestinais mais prevalentes na zona oeste.

A prevalência de indivíduos sem morbidades e com morbidades também foi significativamente $(p=0,003)$ associada à zona geográfica (figura 1). No caso, idosos residentes na zona sul apresentaram freqüência significativamente menor de pouca morbidade (nenhuma ou uma) e maior freqüência de duas morbidades em relação aos idosos das demais zonas. 


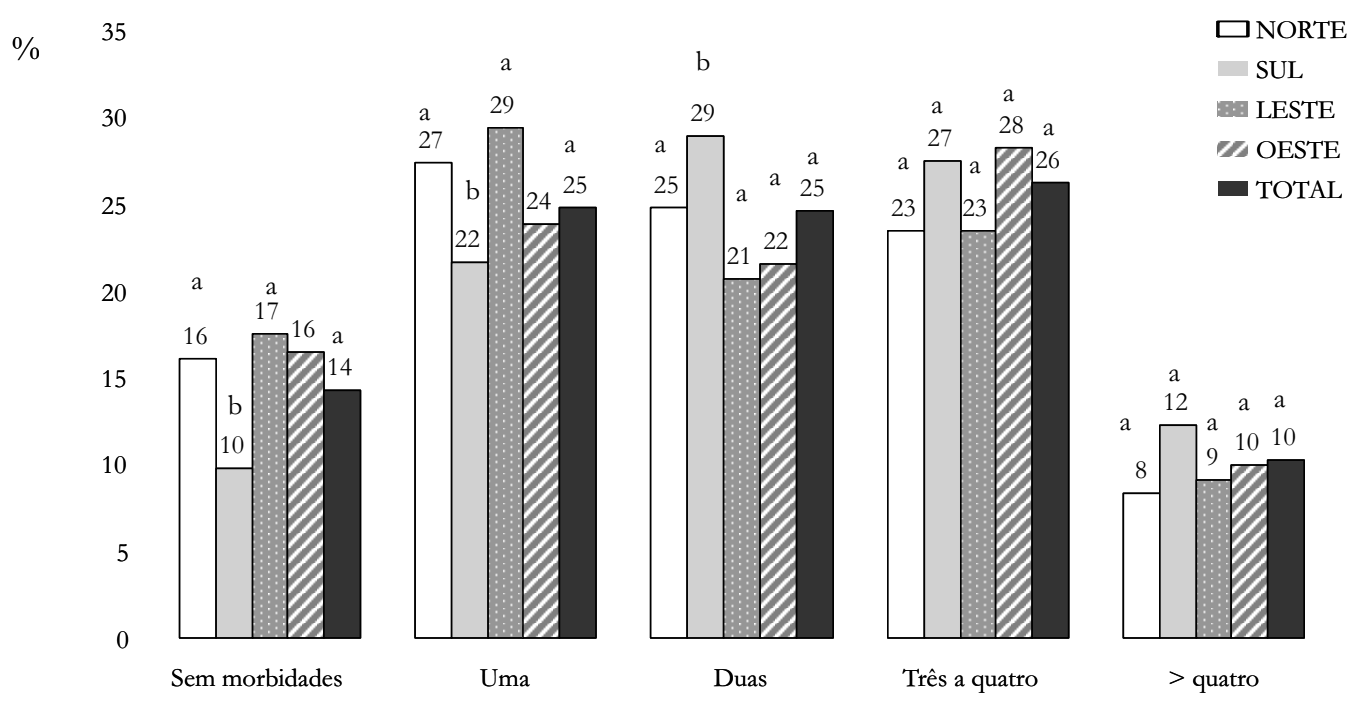

Figura 1 - Distribuição do auto-relato de morbidades crônicas nos idosos inseridos na Estratégia de Saúde da Família (ESF-SUS) do Município de Manaus-AM por zona geográfica das unidades básicas de saúde (novembro de 2007 a maio de 2008). Letras diferentes na mesma categoria de morbidades indicam que não ocorreram diferenças estatisticamente significativas.

\section{DISCUSSÃO}

O estudo aqui apresentado descreveu e comparou indicadores que afetam a saúde e autonomia dos idosos inseridos na ESF-SUS do município de Manaus, e os resultados mostraram variações importantes no quadro da prevalência das variáveis investigadas em relação ao distrito de saúde distribuído por zonas geográficas. Como foram observadas tais diferenças, os resultados serão discutidos sob duas abordagens: (1) as prevalências dos indicadores em relação a outros estudos previamente publicados; e (2) o significado e impacto das diferenças observadas entre os distritos de saúde na saúde pública do idoso de Manaus.

Comparações entre prevalências de estudos populacionais devem ser feitas com mui- to cuidado porque existem os universos populacionais, ou mesmo o delineamento dos estudos pode ser diferente, o que interfere diretamente nos resultados obtidos. Estudos focando idosos na ESF-SUS ainda são bastante incipientes e, por este motivo, a comparação das prevalências entre os indicadores aqui investigados com de outros estudos pode conter vieses relevantes.

Assim, destacada esta limitação, optou-se por comparar os resultados obtidos com os do estudo realizado pelo Serviço Social do Comércio (SESC Nacional, SESC-SP) e a Fundação Perseu Abramo ${ }^{12,13}$ que é referenciado no site do Ministério da Saúde. Tal estudo incluiu 2.136 idosos ( $\geq 60$ anos) em 204 municípios (pequenos, médios e grandes) distribuídos nas cinco macrorregiões do país (Norte, 
Nordeste, Centro-Oeste, Sudeste e Sul) no período de 01 a 23 de abril de 2006 e/ou com dados obtidos nos Censos demográficos do IBGE.

No estudo "Idosos do Brasil"12,13 a amostragem também foi do tipo probabilística nos primeiros estágios (sorteio dos municípios, dos setores censitários e domicílios), combinada com controle de cotas de sexo e idade para seleção do indivíduo (estágio final). Como podemos observar na figura 2 , que apresenta e compara alguns dos indicadores socioeconômicos e demográficos, a maioria foi muito similar entre as duas amostras, indicando que os idosos aqui analisados apresentam, em geral, o padrão socioeconômico e cultural médio do idoso brasileiro. Dentro destes fatores, merece destaque o número elevado de analfabetos funcionais entre os idosos.

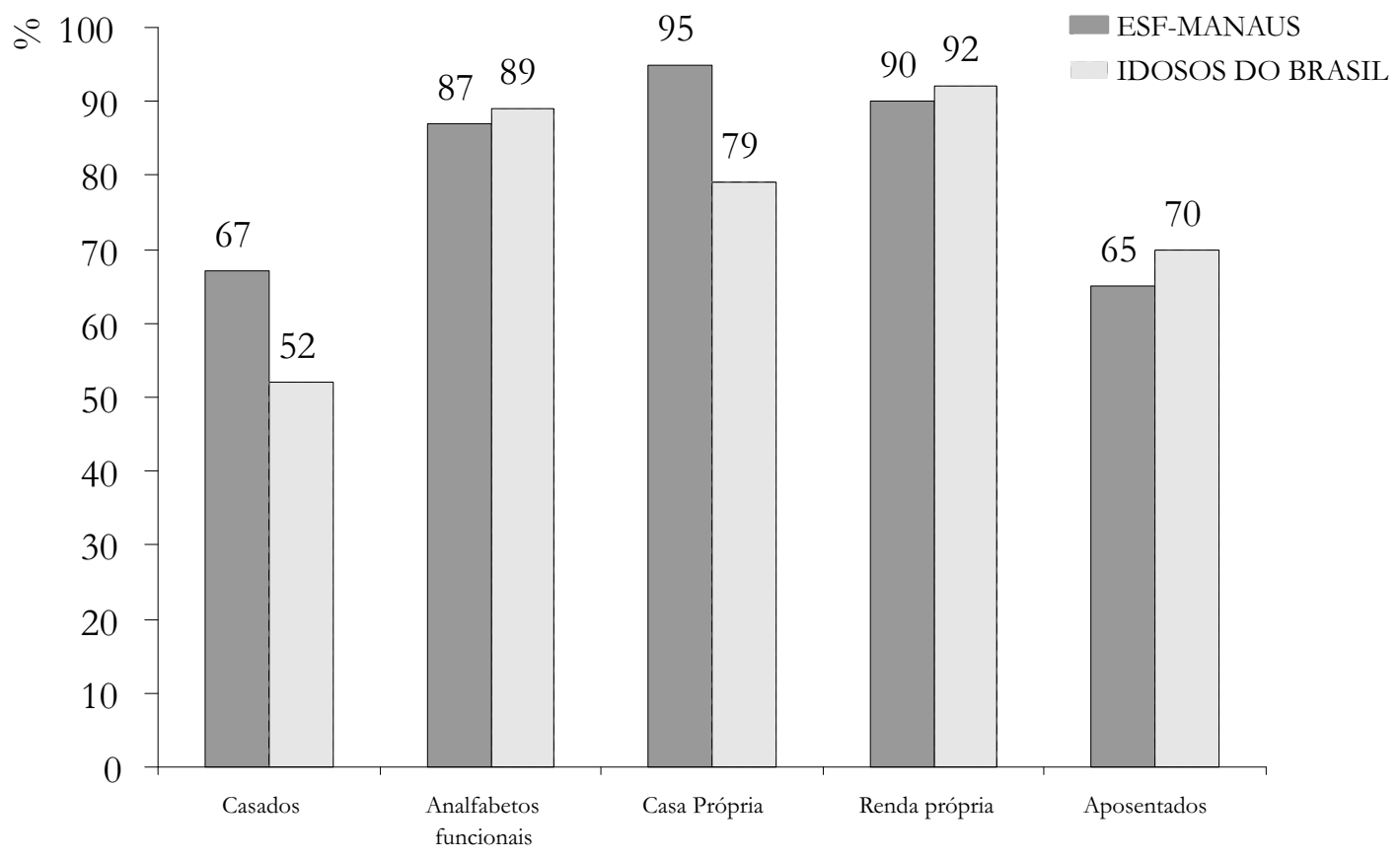

Figura 2 - Comparação de alguns indicadores socioeconômicos e culturais dos idosos participantes do Projeto Idoso da Floresta (ESF-SUS Manaus) e dos idosos incluídos no estudo “Idosos do Brasil”" (Neri, 2007) ${ }^{13}$ 
Ainda que o Censo 2000 (IBGE) tenha descrito que $64,8 \%$ dos idosos brasileiros sabiam pelo menos ler e escrever, valor muito próximo ao observado neste trabalho $(65,9 \%)$, e que em relação ao Censo de 1991 ocorreu crescimento de 16,1\%, ainda existem cerca de 5,1 milhões de idosos analfabetos no Brasil. No universo investigado no presente estudo, 34,1\% estão incluídos nesta categoria.

Os índices são ainda altos e problemáticos, uma vez que existem estudos epidemiológicos que mostram que o analfabetismo ou a baixa escolaridade estão fortemente associados com indicadores de morbidade e dependência em idosos. Estudo transversal conduzido por Rosa et al. ${ }^{14}$ em São Paulo, no ano de 1989, avaliando a capacidade funcional através da escala de atividades de vida diária pessoal e instrumental em 964 idosos, descreveu que idosos com nível mais baixo de escola- rização (apenas lê e escreve/analfabeto) apresentaram cinco vezes mais chance de ter dependência moderada a grave.

Nos indicadores de atenção à saúde, morbidades e autonomia, quando comparamos os resultados, aproximadamente $32 \%$ das mulheres idosas incluídas no estudo "Idosos Brasileiros" relataram fazer exame de mama pelo menos uma vez ao ano, freqüência muito próxima à observada no presente estudo (35,6\%). Já o relato de imunização também mostrou freqüência muito próxima entre o observado em nossa amostra (74\%) e a da Região Norte $(75 \%)$, sendo maior que a do Brasil como um todo (71\%).

A prevalência auto-relatada de algumas morbidades também foi comparada entre os resultados aqui obtidos e os descritos no estudo "Idosos do Brasil". 12,13 Tal comparação é apresentada na figura 3.

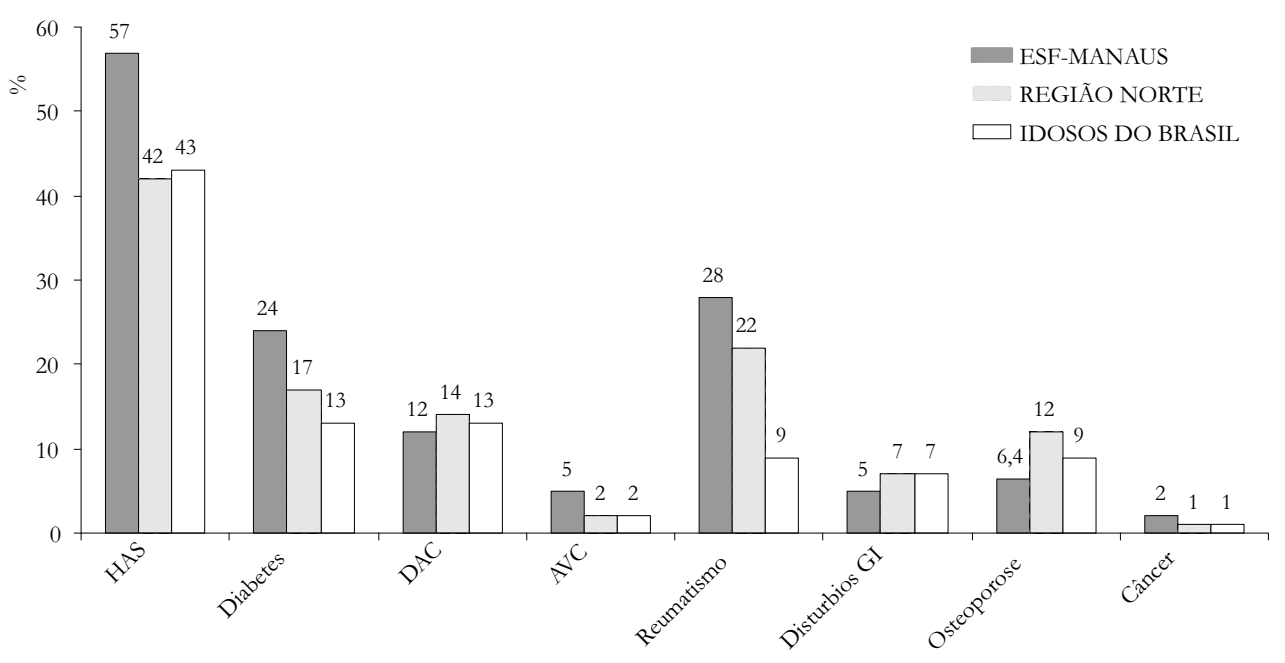

Figura 3 - Comparação da prevalência de morbidades auto-relatadas pelos idosos participantes deste estudo e do estudo "Idosos do Brasil" realizado pelo SESC e Fundação Perseu Abramo (2007) ${ }^{12}$ com uma amostra probabilística de idosos brasileiros das diferentes macro-regiões do País. 
Os resultados mostram um perfil muito próximo entre as duas amostras, com exceção da hipertensão arterial sistêmica e diabetes, que parece ser maior nos idosos aqui analisados. Estas diferenças podem resultar de: (1) um viés de seleção dos indivíduos que acabaram sendo inseridos no ESF-SUS por já estarem sendo atendidos pela rede pública de saúde; (2) ou também por terem sido diagnosticados por ocasião desta inserção. Isto ocorre porque muitos portadores, tanto de hipertensão quanto de diabetes, muitas vezes não têm conhecimento do seu estado de saúde, já que tais morbidades nos estados iniciais não apresentam sintomatologia clínica clara. Deste modo, a comparação entre amostras da população como um todo e amostras da população atendida pela rede pública, institucionalizada ou pertencente a serviços de saúde, pode apresentar diferenças importantes na prevalência de tais morbidades. Mesmo assim, na Região Norte como um todo, o diabetes mellitus apresentou prevalência $5 \%$ maior do que outras regiões brasileiras, fato que deve receber atenção do serviço público de saúde no sentido de entender suas causas e desenvolver mecanismos de prevenção primária desta doença que possui alta carga de morbimortalidade.

Estudo sobre a prevalência do diabetes mellitus realizado pela Coordenação Geral de Agravos e Doenças Não-transmissíveis da Secretaria de Vigilância da Saúde do Ministério da Saúde (2002-2003) $)^{15}$ relatou que, em Manaus, $44,1 \%$ da população entre 25 a 39 anos, $76,3 \%$ da população entre 40 a 59 anos e $82,4 \%$ da população entre 60 anos ou mais referiram ter realizado exame para medir o açúcar no sangue ou diagnosticar diabetes. Manaus também foi a capital que apresentou maior prevalência de diagnóstico clínico do diabetes em relação às outras 14 capitais e ao distrito federal. No caso, a prevalência de idosos diabéticos (> 60 anos) foi de 8,9\% (6,5$11,4)$. O estudo também mostrou que indivíduos com baixa escolaridade eram mais afetados pela doença $(16,7 \%$ em sujeitos com o ensino fundamental incompleto e 3,7\% nos sujeitos com ensino fundamental completo ou mais). Uma vez que a maioria dos idosos aqui investigados possui baixa escolaridade, a alta prevalência de diabetes observada nesta investigação é explicável.

Estudo do tipo inquérito domiciliar no município de Uberaba, comparando as condições de saúde de idosos diabéticos, foi realizado entre dezembro de 2005 a fevereiro de 2006 e incluiu 417 idosos. Os resultados mostraram que a maioria dos idosos portadores da doença tinha de 60 a 70 anos de idade, era do sexo feminino, fazia uso de medicamentos e apresentava os seguintes problemas associados: hipertensão, problemas de visão e de coluna. Além disso, as mulheres diabéticas percebiam sua saúde como má ou péssima e referiam o custo dos medicamentos como o principal problema para sua aquisição. ${ }^{16} \mathrm{Um}$ estudo complementar no Projeto Idoso da Floresta, comparando a prevalência do diabetes mellitus entre homens e mulheres, morbidades associadas e o impacto na qualidade de vida, seria de grande relevância para o ESF-SUS, a fim de caracterizar este subgrupo populacional altamente suscetível à fragilidade. ${ }^{17}$ 
Os autores do estudo conduzido em Uberaba também salientaram a importância de se estabelecer programas de prevenção secundária envolvendo educação em saúde, como o incentivo aos cuidados pessoais, a realização de atividade física moderada e com início gradativo, a ingestão de líquidos; a manutenção, em acesso fácil, de carboidrato de rápida absorção para casos de hiperglicemia, o uso de calçados e meias adequados e o autoexame dos pés.

A ocorrência de polimorbidades e de idosos autônomos/dependentes também apresentou resultados que merecem consideração especial. Ainda que o conceito de capacidade funcional guarde grande complexidade, na prática ele reflete o quanto o idoso é capaz ou incapaz de executar tarefas cotidianas de modo independente. Ou seja, a incapacidade funcional é definida como a dificuldade, devido a uma deficiência, para realizar atividades típicas e pessoalmente desejadas pela sociedade. ${ }^{18}$

No estudo "Idosos do Brasill", 12,13 a maioria relatou possuir pelo menos uma morbidade crônica $(81 \%)$, prevalência esta muito próxima à verificada neste estudo $(86 \%)$. Estudo conduzido em uma área de abrangência da Estratégia de Saúde da Família de um município do norte do Paraná avaliou estas duas variáveis em 86 idosos com 75 anos ou mais. O estudo mostrou que $77,9 \%$ dos idosos eram independentes, ainda que $76,7 \%$ apresentassem co-morbidades. ${ }^{19}$ Outra investigação realizada em 523 idosos de baixa renda do município de São Carlos-SP, cadastrados na Estratégia de Saúde da Família, des- creveu que $72 \%$ eram independentes e $77 \%$ possuíam de uma a cinco enfermidades. ${ }^{20}$

Silvestre \& Costa Neto, ${ }^{21}$ na publicação "Abordagem do idoso em programas de saúde da família", fizeram uma síntese das principais características e demandas básicas associadas à atenção básica da saúde do idoso. Os autores apresentaram prevalências incluindo a de multipatologias, estimadas em 10\%, como observado no presente estudo. A prevalência de hospitalizações também foi similar à aqui descrita (os autores relatam 16\% de hospitalizações).

Em síntese, ainda que a revisão de todos os estudos sobre o perfil de saúde e autonomia do idoso, bem como das morbidades e outros indicadores relacionados, não tenha sido esgotada neste artigo, o conjunto geral dos resultados mostra padrões de prevalência relativamente similares a outros estudos. É claro que o principal fator limitante nestas comparações diz respeito aos delineamentos metodológicos per se e também ao universo da pesquisa, que no caso desta investigação está concentrada no idoso que freqüenta o ESF-SUS de Manaus-AM.

"O aumento dos idosos na população implica, em termos de utilização de serviços de saúde, maior número de problemas de longa duração, que frequentemente exigem intervenções custosas, envolvendo tecnologia complexa para um cuidado adequado". ${ }^{7}$ Em síntese, "qualquer que seja o indicador de saúde analisado, haverá uma proporção maior de agravo e procedimentos médicos entre aqueles de mais de 60 anos (...)". ${ }^{22}$ Nestes termos, conhecer a "epidemiologia do 
idoso" no local da rede básica de saúde é de grande relevância, uma vez que tal conhecimento é importante para se estabelecer programas de prevenção e de assistência o mais realísticos e efetivos possível.

É claro que são necessárias análises adicionais, considerando-se, por exemplo, a influência da idade, do sexo e de outros fatores socioeconômicos e culturais associados à prevalência das morbidades, disfunções e dependência dos idosos. Adicionalmente, é fundamental a investigação de indicadores da qualidade de vida dessa população. Por este motivo, outra limitação do presente estudo é ainda não realizar análises estratificadas por sexo, idade, condição socioeconômica e cultural. Tais análises serão aprofundadas em estudos complementares futuros.

Em relação à segunda abordagem a ser aqui discutida, que versa sobre o significado e o impacto das diferenças observadas entre os distritos de saúde na saúde pública do idoso de Manaus, é importante tecer alguns comentários. Analisando-se os resultados encontrados, podemos dizer que os idosos não apresentaram um perfil homogêneo nos quatro distritos de saúde do município. Na comparação inicial, como era de se esperar, existiram variações socioeconômicas e culturais entre as diferentes zonas geográficas de Manaus. No estudo, a zona leste foi identificada como a que apresenta maior freqüência de idosos solteiros, maior número de idosos com renda muito baixa, com menor educação e com menos assistência externa (auxílios).
Estas condições podem ser explicadas pela origem histórica da zona leste, que surgiu no final da década de 70 com a ocupação de ribeirinhos, moradores de outras cidades e da periferia, apresentando grandes conflitos fundiários nas décadas passadas. Apesar da sua origem, a ocupação da zona leste é diversa, possuindo hoje bairros pobres e ricos. É a região de Manaus que apresenta maior centro comercial da cidade, além de ser a mais populosa e possuir o maior colégio eleitoral do Amazonas e da Amazônia. É na zona leste que se concentram alguns dos bairros mais populosos da cidade. Ao mesmo tempo, possui o maior hospital estadual, o ProntoSocorro João Lúcio Pereira Machado, que atende às principais urgências do Amazonas, e também o Pronto-Socorro da Zona Leste Platão Araújo e a Maternidade Ana Braga.

Por outro lado, o distrito de saúde da zona sul foi o que apresentou o melhor conjunto de indicadores socioculturais e econômicos. A zona sul concentra a grande maioria dos bairros antigos de Manaus, sendo que muitos são menos povoados. Neste local se encontram as fábricas e o distrito industrial. Alguns bairros, como Educandos e Aparecida, possuem bons índices sociais, se comparados a outros bairros do município, sendo seu maior problema os índices altos de poluição em seus igarapés e lagoas. Também abriga o bairro Cachoeirinha, considerado um dos mais seguros para se viver e que também possui condições sociais e um bom sistema de transporte coletivo. Por outro lado, o distrito de saúde da zona sul também possui bairros com problemas sociais e falta de segurança, como é o caso do bairro Colônia Oliveira Machado. 
Apesar destas diferenças socioeconômicas e culturais que influenciam a saúde e a qualidade de vida do idoso, a prevalência de idosos independentes e dependentes foi similar em todos os distritos, enquanto que os indicadores de saúde necessariamente não foram piores na zona leste em relação às demais zonas. Deste modo, as diferenças observadas entre as regiões precisam ser avaliadas possivelmente sob o olhar dos profissionais que atendem o ESF-SUS da região, a fim de se estabelecer ações efetivas associadas aos resultados obtidos. Estudos adicionais estratificando sexo, idade, perfil socioeconômico e cultural dos indicadores que apresentaram resultados diferenciados segundo o distrito de saúde deverão ser feitos com o intuito de se identificar os subgrupos populacionais mais fragilizados e se estabelecer ações preventivas mais eficazes e baseadas na realidade local.

Adicionalmente, os resultados obtidos servem como referência que permitirá uma análise prospectiva das ações do ESF-SUS de Manaus-AM no atendimento dos idosos. É claro que muitos percalços existirão se considerarmos que "pelo fato de lidarmos com um grupo etário que tem crescido muito em anos recentes, existe uma enorme carência de profissionais treinados, com formação específica e cursos reconbecidos pela qualidade acadêmica." ${ }^{22} \mathrm{E}$ a fim de atender às demandas existentes, foram implantadas estratégias de formação de recursos humanos dos serviços municipal e estadual de saúde de Manaus junto à Universidade Aberta da Terceira Idade da Universidade do Estado do Amazonas (UnATI-UEA). Estima-se que a formação deste quadro profissional tenha impacto significativo e profundo no cuidado do idoso.
Além do mais, a formação desses recursos humanos vai ao encontro do referencial de atenção preconizado pela Estratégia de Saúde da Família. No contexto da ESF-SUS, “destaca-se o trabalho dos profissionais de saúde voltado para a assistência integral e continua de todos os membros das famílias vinculadas, em cada uma das fases do seu ciclo de vida, sem perder de vista seu contexto familiar e social." ${ }^{21}$ Em relação à promoção de saúde do idoso, é de suma importância a capacidade da equipe de saúde de identificar fatores determinantes na qualidade de vida, no seu contexto familiar e social, para além dos cuidados e controle das morbidades e disfunções.

Em síntese, o estudo aqui descrito apresenta indicadores gerais de saúde dos idosos inseridos no ESF-SUS do município de Manaus-AM. Os resultados sugerem que o perfil socioeconômico e cultural, bem como da prevalência de morbidades e dependência, são aproximadamente similares a de outros locais do país. Entretanto, existem variações importantes entre os quatro distritos de saúde do município de Manaus que devem ser consideradas na construção do perfil epidemiológico do idoso e nas estratégias de atenção associadas a este grupo etário e mais bem analisadas em estudos complementares que estratifiquem a amostra por sexo, idade e perfil socioeconômico e cultural.

\section{NOTAS}

Autor principal do Projeto Idoso da Floresta. Doutor. E-mail: eer@vivax.com.br

Colaboradora no delineamento, na coleta das informações e na discussão dos resultados. Especialista. E-mail: edneia@vivax.com.br 
Universidade Aberta da Terceira Idade, Universidade Estadual do Amazonas

Av. Djalma Batista, 2470. 69050-900 - Manaus, AM, Brasil

b Colaborador no delineamento do estudo, organização e revisão final do manuscrito. Doutor. E-mail: veras@uerj.br

d Colaboradora na revisão teórica e final do manuscrito. Doutor. E-mail: celpcaldas@hotmail.com

Universidade do Estado do Rio de Janeiro, Universidade Aberta da Terceira Idade - UnATI, Rua São Francisco Xavier, 524, $10^{\circ}$ andar. 20559-900 - Rio de Janeiro, RJ, Brasil

c Colaboradora no delineamento do estudo, organização do instrumento de avaliação do idoso, e na revisão fina do manuscrito. Mestre. E-mail: karin_viegas@pucrs b Pontifícia Universidade Católica do Rio Grande do Sul, Faculdade de Enfermagem Nutrição e Fisioterapia, Programa de Pós-Graduação em Gerontologia Biomédica. Av. Ipiranga, 6681 Prédio 12. 90619-900 - Porto Alegre, RS, Brasil

f Colaboradora na organização do banco de dados, análise estatística dos resultados e redação do manuscrito). Doutor. Email: bebelugalde@hotmail.com g Colaboradora no delineamento, análise dos dados e redação do manuscrito. Doutor. E-mail: ibmcruz@hotmail.com

Universidade Federal de Santa Maria, Laboratório de Biogenômica, Departamento de Morfologia, Centro de Ciências da Saúde.

Av. Roraima 1000, Prédio 19, Sala 3126. 97105-900 - Santa Maria, RS, Brasil

\section{AGRADECIMENTOS}

Os autores agradecem a todos os alunos do Curso de Especialização em Gerontologia da Universidade Aberta da Terceira Idade da Universidade do Estado do Amazonas (UnATI-UEA), bem como aos funcionários da UnATI-UEA, pelo auxílio na coleta dos dados e na logística de execução do projeto.

5. Motta LB, Aguiar AC. Novas competências profissionais em saúde e o envelhecimento populacional brasileiro; integralidade, interdisciplinariedade e intersetorialidade. Ciência Saúde Coletiva 2007, 12: 363-72.

6. Silvestre JA. Capacitação em saúde do idoso. Brasília, DF: Ministério da Saúde. 1999.

7. Veras RP et al. A assistência suplementar de saúde e seus projetos de cuidado para com o idoso. Ciência Saúde Coletiva 2008, 13: 119-26.

8. Lima-Costa MF, Veras RP. Saúde pública e envelhecimento. Cad Saúde Pública 2008, 19:

9. Souza HM. Saúde da Família: desafios e conquistas. In: Negri B, Viana AL, (organizadores). O Sistema Único de Saúde em dez anos de desafios. São Paulo: Sociedade Brasileira de Vigilância em Medicamentos/ Centro de Estudos Augusto Leopoldo Ayrosa Galvão; 2002. p. 221-40. 
10. Escorel S, Giovanella L, Mendonça MHM, Senna MCM. O Programa de Saúde da Família e a construção de um novo modelo para a atenção básica no Brasil. Rev Panam Salud Publica 2007, 21: 164-76.

11. Araújo F, Ribeiro JLP, Oliveira A, Pinto C. Validação do Índice de Barthel numa amostra de idosos não institucionalizados. [acesso em: 2008 ago.10). Disponível em: URL: http://www.ensp.unl.pt/ dispositivos-de-apoio/cdi/cdi/sector-depublicacoes/revista/2000-2008/pdfs/ 05_02_2007.pdf

12. Fundação Perseu Abramo. Idosos no Brasil : Vivências, desafios e expectativas na $3^{\mathrm{a}}$ idade. [acesso em: 2008 ago 10). Disponível em: URL: http://www2.fpa.org.br/portal/ modules $/$ news $/$ index.php? storytopic $=1642$

13. Neri AL. Idosos no Brasil: vivências, desafios e expectativas na terceira idade. São Paulo: SESC SP; 2007.288p.

14. Rosa TEC, Benicio MHD, Latorre MRDO. Fatores determinantes da capacidade funcional entre idosos. Rev Saude Publica 2003, 37: 40-8.

15. Instituto Nacional de Câncer (Brasil). Inquérito domiciliar sobre comportamento de riscos e agravos não transmissíveis. Brasil, 15 capitais e Distrito Federal, 2002-2003. [acesso em: 2008 ago10). Disponível em: URL: http:/ / www.inca.gov.br/inquerito/docs/diabete.pdf
16. Tavares DMS, Pereira GA, Iwamoto HH, Miranzzi SSC, Rodrigues LR, Machado ARM. Incapacidade Funcional entre idosos residentes em um município do interior de Minas Gerais. Texto \& contexto enfermagem / UFSC 2007, 16: 32-9.

17. Saydah SH, Eberhardt MS, Loria CM, Brancati FL. Age and burden of death attributable to diabetes in the United States. Am J Epidem 2002, 156: 714-9.

18. Verbrugge LM, Jette AM. The disablement process. Soc Sci Med 1994, 38:1-14.

19. Farinasso ALC, Marques S, Rodrigues RAP, Hass VJ. Capacidade funcional e morbidades referidas de idosos em uma área de abrangência do PSF. Rev Gaucha Enferm 2006, 27: 45-52.

20. Feliciano AB, Moares AS, Freitas ICM. O perfil do idoso de baixa renda no Município de São Carlos, São Paulo, Brasil: um estudo epidemiológico. Cad Saude Publica 2004, 20:1575-85.

21. Silvestre JA, Costa Neto MM. Abordagem do idoso em programas de saúde da família. Cad Saude Publica 2003; 19: 839-47.

22. Veras RP. Em busca de uma assistência adequada à saúde do idoso: revisão da literatura e aplicação de instrumentos de detecção precoce e de previsibilidade de agravos. Cad Saude Publica 2003, 19:705-15. 